\title{
Mouse Behavior Tracker: An economical method for tracking behavior in home cages
}

\author{
Sudheer K. Tungtur ${ }^{1}$, Natsuko Nishimune ${ }^{1}$, Jeff Radel ${ }^{2}$, and Hiroshi Nishimune ${ }^{1 *}$ \\ ${ }^{1}$ Department of Anatomy and Cell Biology, University of Kansas School of Medicine, Kansas City, KS and \\ ${ }^{2}$ Department of Occupational Therapy Education, University of Kansas School of Health Professions, \\ Kansas City, KS
}

BioTechniques 63:215-220 (November 2017) doi 10.2144/000114607

Keywords: ImageJ, mouse behavior, distance traveled, velocity, SOD1 ${ }^{\text {G93A }}$

Supplementary material for this article is available at www.BioTechniques.com/article/114607.

Analysis of mouse behavior often requires expensive equipment and transfer of the mice to new test environments, which could trigger confounding behavior alterations. Here, we describe a system for tracking mouse behavior in home cages using a low-cost USB webcam and free software (Fiji and wrMTrck). We demonstrate the effectiveness of this method by tracking differences in distance traveled, speed, and movement tracks between wild-type mice and amyotrophic lateral sclerosis (ALS) model mice (SOD1 ${ }^{\mathrm{G} 93 \mathrm{~A}}$ ).

Precise and accurate behavior analyses are essential for phenotyping diseasemodel mice and genetically modified mice (1). Although methods exist for measuring spontaneous behaviors of mice (actometers, running wheels, etc.), methods for quantifying distance traveled and tracking movement patterns in a home cage are limited. For example, running wheels allow measurement of the distance traveled in a home cage, but the results are complicated by the exercise effect of the activity in the wheel.

Commercial systems and custom systems can measure the distance traveled inside a cage and analyze mouse behaviors (Supplementary Table S1) (2-10); however, these systems use specialized cages, requiring animals to be transferred to a new environment for analysis. For some strains, altered behavior has been observed even after 3 weeks of habituation to a commercial metabolic cage system (11). Some commercial systems can analyze behavior in home cages, but one such system does not measure distance traveled (HomeCageScan; CleverSys Inc, Reston, VA), and another system monitors activity but does not accurately measure distance traveled (Infrared Motion Detector; Starr Life Sciences Corp., Oakmont, PA).

Here, we address these limitations by developing a system to track mouse behavior using free public domain software for video tracking: wrMTrck and Fiji. wrMTrck was developed to track the behavior of Caenorhabditis elegans and has also been applied successfully to track melanoblasts, mitochondria, Schistosoma mansoni, Xenopus tropicalis tadpoles, and yeast (12-17). Our method, which we named Mouse Behavior Tracker, measures distance traveled, average speed, and movement patterns/ tracks in home cages using economical USB-webcams, free software, and a standard desktop computer. When Mouse Behavior Tracker results were compared with a commercial system that uses a video tracking method, EthoVision XT (Noldus Information Technology Inc., Leesburg, VA), very similar results were obtained.

\section{Materials and methods \\ Animal models}

Animal experiments were performed in accordance with the animal care and use protocol approved by the Institutional Animal Care and Use Committee of the University of Kansas Medical Center and its Guidelines for the Care and Use of Laboratory Animals. Three to six mice were analyzed for each genotype or coat color. Mice with black coat color-C57BL/6J wild-type male mice (JAX Stock No: 000664) and SOD1 ${ }^{\text {G93A }}$ male mice [JAX Stock No: 004435; B6.Cg-Tg(SOD1*G93A) 1 Gur/J, high-copy number line on a pure B6 background]-were purchased from the Jackson Laboratory (JAX, Bar Harbor, ME) and analyzed in this study. Mice with brown coat colorRosa tdTomato-EGFP, Wnt1-Cre male mice [Gt(ROSA)26Sor_tm4(ACTBtdTomato,-EGFP)Luo_J (JAX Stock No: 007576) crossed with Tg(Wnt1cre)11Rth Tg(Wnt1-GAL4)11Rth_J (JAX Stock No: 003829)]-and a littermate wild-type female mouse were analyzed in this study. White coat color mice-two female wild-type mice-were analyzed in this study. The mice used in this study had been bred in our institutional animal facility, kept on a $14 \mathrm{~h}$ light/10 h dark (6:00 am to 8:00 pm) cycle, and fed ad libitum with normal chow (53WU,

\section{METHOD SUMMARY}

Here, we present a new method for tracking mouse behavior in home cages. Our approach allows quantification of distance traveled, average speed, and movement tracks using a significantly lower-cost system compared with commercial alternatives. 
PicoLab Rodent Diet 20; LabDiet, St. Louis, MO). No statistical methods were used to predetermine sample size. A method of randomization was not used for comparisons of disease-model to wild-type mice because the animals were separated by genotype.

\section{Video recording}

Mouse behavior was recorded using an economical webcam (Creative WebCam Instant Model VF 0040; Creative Technologies Ltd. Jurong East, Singapore) with the following settings: $320 \times 240$ pixels, 30 frames per second (fps), MJPEG compressor. Recordings were done between 12:00 PM and 4:00 PM under room ceiling fluorescent lighting as 20-min videos.

Tracking mouse behavior using Fiji The analysis protocol described here was developed for macOS 10.12 using Fiji (version 1.51h, https://fiji.sc/) running on a MacBook Pro (Apple Inc., Cupertino, CA) (CPU: Intel Core i7 $2.8 \mathrm{GHz}$; flash storage: $751 \mathrm{~GB}$; RAM: 16GB 1.6 GHz DDR3; graphics: NVIDIA GeForce GT 650M $1024 \mathrm{MB}$; operating system: macOS 10.12.6). The RAM size of $16 \mathrm{~GB}$ was sufficient to process 30-fps videos up to 30-min long. The combination of Fiji, wrMTrck plugin, and the Mouse Behavior Tracker macro was confirmed to function properly on Mac OsX 10.9, 10.11, and macOS 10.12. Mouse behavior was tracked using the publicly available free Fiji software, the wrMTrck plugin for ImageJ (wrmtrck.zip developed by Jesper Søndergaard Pedersen; www. phage.dk/plugins/wrmtrck.html), and our Mouse Behavior Tracker macro. The Mouse Behavior Tracker source codes for the AVI (MJPEG) file format and MP4 file format are included with the protocol in the Supplementary Material. The same video data files were analyzed using EthoVision XT (Noldus
A

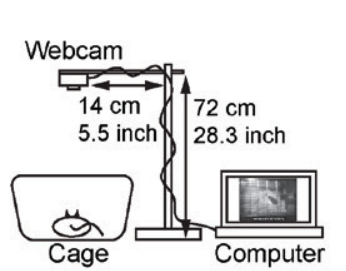

E

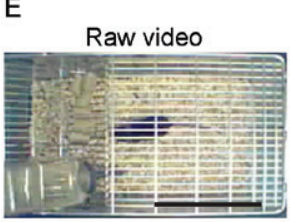

$\mathrm{F}$

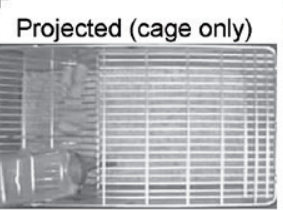

C

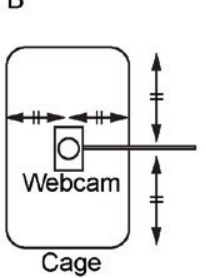

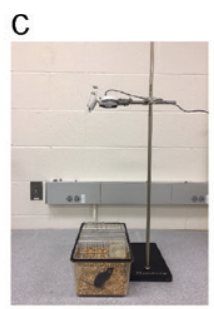

G

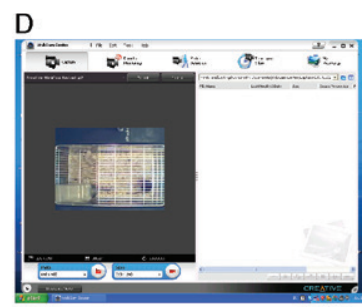

$\mathrm{H}$

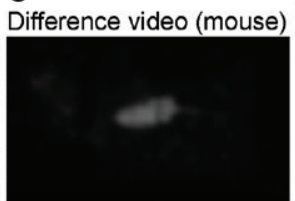

Tracking video

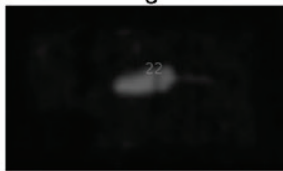

Figure 1. The Mouse Behavior Tracker method. Schematic diagram of the video recording setup showing the side view $(A)$ and the top-down view (B), with dimensions. (C) A mouse being recorded by a Creative WebCam Instant Model VF 0040. (D) A screenshot of the Creative camera software showing a recording of a mouse. Representative images are shown from a raw video of a C57BL/6J wild-type mouse recorded at 30 frames per second (fps) (E), an average intensity-projected image showing the cage without the mouse (F), the differential binary-image video showing the mouse after subtracting the projected empty cage image from the raw video $(\mathrm{G})$, the tracking video showing the mouse with a tracking number assigned by Mouse Behavior Tracker $(\mathrm{H})$, and movement tracks of the mouse for a 1-min period (I). Scale bar in (E): $10 \mathrm{~cm}$.

Information Technology Inc., Leesburg, VA) for validation.

\section{Statistics}

All statistics were performed using GraphPad Prism software version 6 (GraphPad Software, Inc., La Jolla, CA). Significance was assessed by an unpaired $t$-test with a two-tailed $P$ value. The $P$ value and the $n$ value are described in the text, and the data are shown as mean \pm SEM

A detailed protocol is available in the online Supplementary Material.

\section{Results and discussion}

Mouse behavior was recorded in a home cage with an inner lid, without the filter tops, using a webcam positioned above the cage on a chromatography column stand with adjustable clamps. An economical USB webcam provided sufficient sensitivity, resolution, and frame rate (Creative

live webcam instant VF 0040, $320 \times 240$ pixels, $30 \mathrm{fps}$ ) to record mouse behavior in the home cage (Figure 1, Supplementary Video S1). File sizes for 20-min videos averaged $123 \mathrm{MB}$. Therefore, a desktop computer (Dell Precision PWS390; Dell, Inc., Round Rock, TX) with standard hard disk storage space was sufficient for the recording (CPU: Intel Core2 Duo 6600, $2.4 \mathrm{GHz}$; RAM: $2 \mathrm{~GB} 2.3 \mathrm{GHz}$; internal hard disk: $148 \mathrm{~GB}$; operating system: Windows XP Professional Version 2002 Service Pack 3).

The 20-min videos were analyzed using Fiji (ImageJ package software) running on an iMac 14.2 (Apple Inc.) with RAM size increased to $24 \mathrm{~GB}$ (CPU: Intel Core i5, 3.2 GHz; hard disk size: $1 \mathrm{~TB}$; RAM: 24GB, $1.6 \mathrm{GHz}$ DDR3; operating system: Mac OsX 10.9.5). Videos were Z-projected in Fiji using the "Average Intensity Type" to generate an image of the cage without a mouse (Figure 1F). This cage image was subtracted from each

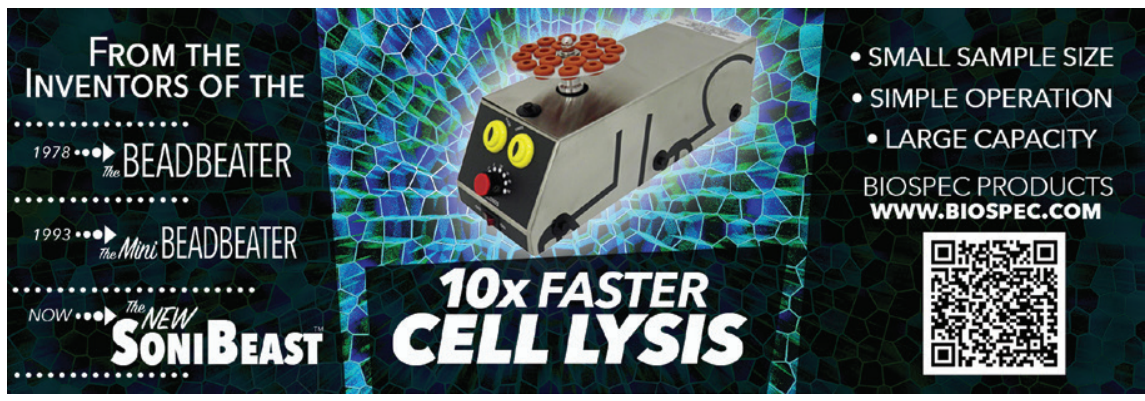
frame of the 20-min video to generate a video of a differential binary image of mouse behavior without the cage (Figure 1G). The differential binary image video was processed using the "Gaussian Blur" filter to remove shadows resulting from the wire lid of the cage above the animal. Resulting time-lapse video was thresholded and tracked using Fiji and the wrMTrck plugin for ImageJ. The wrMTrck plugin is based on the Object Tracker 
plugin developed by Wayne Rasband, who developed ImageJ at the $\mathrm{NIH}$. The two plugins use the Pythagorean theorem to calculate the distance traveled by a tracked object. The 20-min video was trimmed down to $1 \mathrm{~min}$ to show representative results of a movement track and a tracking video (Figure 1H, Supplementary Video S2). A representative result of a movement track generated from a 20-min video is shown in Figure 3C. These results suggest that Mouse Behavior Tracker is capable of tracking mouse behavior in videos ranging from 1 min to 20 min. A Fiji macro was created for these processes (described in the Supplementary Material). Mouse Behavior Tracker generates the following data: (i) tracking data (distance traveled and average speed) as raw and summarized data in Microsoft Excel files, (ii) video of mouse tracking with the tracking object number on the tracked mouse, and (iii) movement tracks (Figure 1I). Mouse Behavior Tracker gave identical results when the same video of a mouse with a black coat color was analyzed three times. Thus, Mouse Behavior Tracker allowed us to quantify mouse activity in a home cage.

One commercial system, EthoVision $\mathrm{XT}$, uses video tracking methods and quantifies distance traveled in home cages. Therefore, we compared Mouse Behavior Tracker and EthoVision XT by analyzing distance traveled in the same video using the two programs. The distance traveled results were very similar, with the ratio of the results obtained from the 2 programs (EthoVision XT result divided by Mouse Behavior Tracker result) being $0.98 \pm 0.03$ (mean $\pm \mathrm{SEM}, n$ = 6 wild-type C57BL/6J mice) (Figure 2); this demonstrates the accuracy of Mouse Behavior Tracker for distance traveled measurements.

We also analyzed mice with brown or white color coats using these two software programs. Both programs could track mice with brown coats. Mouse Behavior Tracker gave identical results when the same video of a mouse with brown coat color was analyzed three times. However, the EthoVision XT quantified 20\% less distance traveled compared with the Mouse Behavior Tracker results using the settings for tracking mice with black coat color. The ratio of the results obtained from EthoVision XT compared

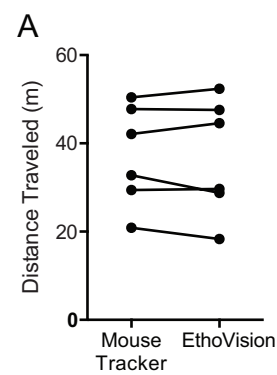

B

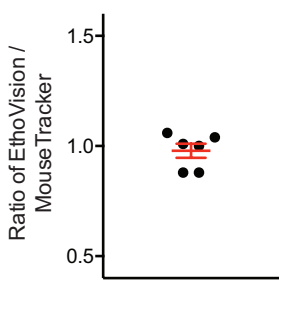

Figure 2. Evaluation of Mouse Behavior Tracker method. The distance traveled by mice in 20 -min-long videos recorded at 30 frames-persecond (fps) was analyzed using the Mouse Behavior Tracker macro or the EthoVision XT software. (A) The graph shows the distance traveled by C57BL/6J wild-type mice, and each line represents one video analyzed using the two programs. (B) The distance-traveled ratio was obtained by dividing EthoVision XT results by Mouse Behavior Tracker results. The graph shows mean \pm SEM (red whiskers). Very similar results were obtained using the two programs.

to Mouse Behavior Tracker was $0.71 \pm 0.1$ (mean \pm SEM, $n=3$ mice). The difference in distance traveled quantified by the two software programs requires further investigation. Mice with white coats could not be tracked by either Mouse Behavior Tracker or EthoVision XT using the settings that track mice with black coats. The inability to track white mice is likely due to the reduced contrast between the white coat color and the corn cob bedding in the cages.

Finally, Mouse Behavior Tracker was used to analyze the behavior of amyotrophic lateral sclerosis (ALS) model mice (SOD1G93A; 139-155 days old) and age- and sex-matched wild-type controls (C57BL/6J; 141-168 days old) in their home cages. The mean distance traveled during a 20-min recording was $13.79 \pm 2.03 \mathrm{~m}$ (mean $\pm \mathrm{SEM}, n=5$ mice) for symptomatic-stage SOD1 ${ }^{\mathrm{G} 93 \mathrm{~A}}$ mice. However, wild-type mice traveled a significantly longer distance of $37.24 \pm 4.68 \mathrm{~m}$ ( $n=6$ mice; $P=0.0021$, unpaired $t$-test) (Figure 3A). In addition, the average speed of SOD1 1993A mice was also significantly slower than that of wild-type mice (SOD1G93A: $1.86 \pm 0.07 \mathrm{~cm} / \mathrm{s}, n=5$ mice; wild-type: $3.49 \pm 0.43 \mathrm{~cm} / \mathrm{s}, n=6$ mice; $P=0.0076$, unpaired $t$-test) (Figure 3B). Furthermore, the movement pattern reflected behavior differences between the two mouse strains. SOD1 ${ }^{\text {G93A }}$ mice moved slowly with alternating lateral displacements of the body, which were represented by characteristic zigzag tracks at the 30 -fps video sampling rate
A

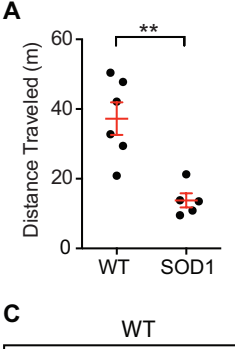

B
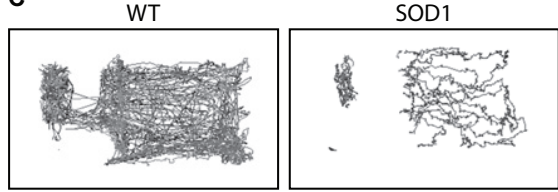

D
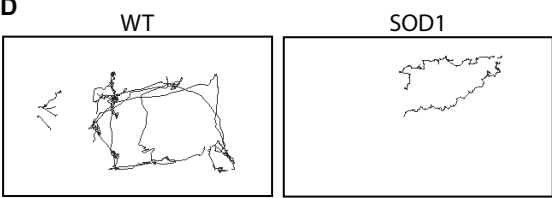

Figure 3. Amyotrophic lateral sclerosis (ALS) model SOD1 ${ }^{693 A}$ mice are less active than wild-type mice and show tottering behavior. Graphs show distance traveled (A) and average speed (B) during the 20 min of recording time. Symptomatic-stage SOD1 ${ }^{\mathrm{G} 93 \mathrm{~A}}$ male mice (SOD1 139-155 days old) traveled a shorter distance at a slower speed than age- and sex-matched wild-type mice (WT, 141-168 days old). A total of $n=5-6$ mice were analyzed. The graph shows mean \pm SEM (red whiskers), and each dot represents one mouse. Asterisks indicate significance by an unpaired $t$-test $(P<0.01)$. (C) Representative movement tracks indicate a higher activity level for wild-type mice than SOD1 $1^{\text {G93A }}$ mice during the 20 -min recordings at 30 frames per second (fps). (D) The tracks of a wild-type mouse show a smooth and balanced movement pattern during a 1-min period; however, the tracks of a SOD1 ${ }^{\mathrm{G} 93 \mathrm{~A}}$ mouse indicated tottering behavior with a zigzag pattern during the same amount of time. These tracks are portions of the tracks shown in (C).

(Figure 3D, Supplementary Videos S3 and S4). This tottering movement of SOD1 ${ }^{\text {G93A }}$ mice may be related to gait differences reported previously for this ALS animal model (18-21). Meanwhile, wild-type mice moved quickly and in a balanced manner, yielding smooth tracks without zigzag tracks. These results demonstrated that there are differences in the activity levels (distance traveled and average speed) and movement patterns between SOD1 ${ }^{\text {G93A }}$ mice and wild-type mice in their home cages.

Measurement of spontaneous activity in non-exercised control animals is seldom undertaken; however, it is essential to compare activity of sedentary animals in home cages with exercised animals with running wheels or forced exercise activities. This is partly due to a lack of simple and economical methods for measuring 
the distance traveled of sedentary animals in their home cages. Systems to analyze home-cage mouse behavior have been developed to quantify behavioral phenotypes (4) and social interactions (7), and mouse behavior has been analyzed using time-lapse VCR systems $(22,23)$; however, these systems do not measure the distance traveled in home cages. Mouse Behavior Tracker is an efficient and low-cost method for measuring distance traveled and movement speed, as well as displaying movement tracks in home cages. Furthermore, this system generates tracks for detecting abnormal behavior, including tottering. Finally, home cage-based analysis is relevant for studies of aging, disease models, genetic manipulation, and pharmacological treatments. It should be noted that for long-term observation over many days or weeks, a commercially available system would be more suitable.

\section{Author contributions}

H.N. designed the experiment. S.T., N.N., and H.N. performed the experiments and analyzed the data. J.R. contributed essential technical expertise on video recording methods. S.T. and H.N. wrote the manuscript, and all authors reviewed the manuscript.

\section{Acknowledgments}

We thank the following software developers for their work and for making their software publicly available: Jesper Søndergaard Pedersen for wrMTrck and the Fiji development team (24). We thank Robert. S. Rogers for technical assistance, Irfan Saadi for the resources, Kenneth McCarson and Michelle Winter for KUMC Rodent Behavior Facility support (NICHD HD 02528). This work was supported by grants from the NIH 1R01NS078214 and 1R01AG051470 (H.N.). This paper is subject to the NIH Public Access Policy.

\section{Competing interests}

The authors declare no competing interests.

\section{Reference}

1. Fonio, E., I. Golani, and Y. Benjamini. 2012. Measuring behavior of animal models: faults and remedies. Nat. Methods 9:1167-1170.
2. Pan, W.H., C.R. Lee, and L.H. Lim. 1996 A new video path analyzer to monitor trave distance, rearing, and stereotypic movement of rats. J. Neurosci. Methods 70:39-43.

3. Quinn, L.P., T.O. Stean, B. Trail, M.S. Duxon, S.C. Stratton, A. Billinton, and N. Upton. 2003. LABORAS: Initial pharmacological validation of a system allowing continuous monitoring of laboratory rodent behaviour. J. Neurosci. Methods 130:83-92.

4. Jhuang, H., E. Garrote, J. Mutch, X. Yu, V. Khilnani, T. Poggio, A.D. Steele, and T. Serre. 2010. Automated home-cage behavioural phenotyping of mice. Nat. Commun. 1:68.

5. Ou-Yang, T.H., M.L. Tsai, C.T. Yen, and T.T. Lin. 2011. An infrared range camera-based approach for three-dimensional locomotion tracking and pose reconstruction in a rodent. J. Neurosci. Methods 201:116-123.

6. Crispim, C.F., Jr., C.N. Pederiva, R.C. Bose, V.A. Garcia, C. Lino-de-Oliveira, and J. Marino-Neto. 2012. ETHOWATCHER: validation of a tool for behavioral and videotracking analysis in laboratory animals. Comput. Biol. Med. 42:257-264.

7. de Chaumont, F., R.D. Coura, P. Serreau, A. Cressant, J. Chabout, S. Granon, and J.C. Olivo-Marin. 2012. Computerized video analysis of social interactions in mice. Nat. Methods 9:410-417.

8. Alexandrov, V., D. Brunner, T. Hanania, and E. Leahy. 2015. High-throughput analysis of behavior for drug discovery. Eur. J. Pharmacol. 750:82-89.

9. Samson, A.L., L. Ju, H. Ah Kim, S.R. Zhang, J.A. Lee, S.A. Sturgeon, C.G. Sobey, S.P.

\section{RAMP-UP}

\section{cell-based assays!}

\section{CLARIOstar ${ }^{\circledR}$ with $\mathrm{ACU}$}

Full flexibility in cellular assays with the new gas ramping function

For the first time scientists are given the capability to fully manipulate the environment within the microplate reader, by mimicking in vitro hypoxia, ischaemia/reperfusion and much more.

The CLARIOstar with LVF Monochromators ${ }^{T M}$ is not only the most-sensitive monochromator-based plate reader today. Its Atmospheric Control Unit (ACU) can run gas ramps, modifying $\mathrm{O}_{2}$ and $\mathrm{CO}_{2}$ gas levels and rapidly restoring back atmospheric conditions.

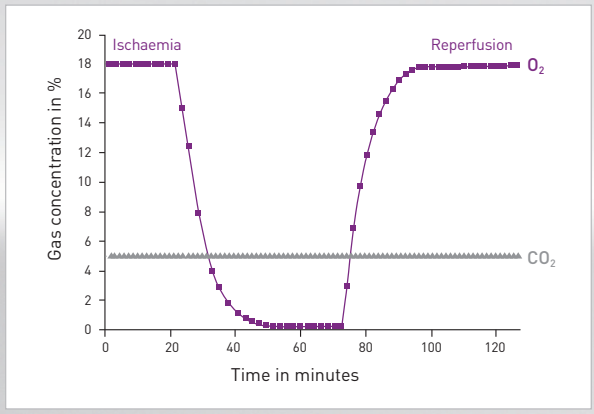

Example of $\mathrm{O}_{2}$ deprivation and reoxygenation (down to $0.2 \% \mathrm{O}_{2}$; purple) with steady $5 \% \mathrm{CO}_{2}$ (grey) performed by the CLARIOstar with $A C U$. 
Jackson, and S.M. Schoenwaelder. 2015. MouseMove: an open source program for semi-automated analysis of movement and cognitive testing in rodents. Sci. Rep. 5:16171.

10. Hewitt, B.M., M.H. Yap, E.F. Hodson-Tole, A.J. Kennerley, P.S. Sharp, and R.A. Grant. 2017. A novel automated rodent tracker (ART), demonstrated in a mouse model of amyotrophic lateral sclerosis. J. Neurosci. Methods. (In press.)

11. Kalliokoski, O., K.R. Jacobsen, H.S. Darusman, T. Henriksen, A. Weimann, H.E. Poulsen, J. Hau, and K.S. Abelson. 2013. Mice do not habituate to metabolism cage housing--a three week study of male BALB/c mice. PLoS One 8:e58460.

12. Beam, M., M.C. Silva, and R.I. Morimoto. 2012. Dynamic imaging by fluorescence correlation spectroscopy identifies diverse populations of polyglutamine oligomers formed in vivo. J. Biol. Chem. 287:2613626145

13. Mort, R.L., M. Keighren, L. Hay, and I.J. Jackson. 2014. Ex vivo culture of mouse embryonic skin and live-imaging of melanoblast migration. J. Vis. Exp. (87)

14. Bros, H., A. Hauser, F. Paul, R. Niesner, and C. Infante-Duarte. 2015. Assessing Mitochondrial Movement Within Neurons: Manual Versus Automated Tracking Methods. Traffic 16:906-917.

15. de Saram, P.S., M. Ressurreicao, A.J. Davies, D. Rollinson, A.M. Emery, and A.J. Walker. 2013. Functional mapping of protein kinase $A$ reveals its importance in adult Schistosoma mansoni motor activity. PLoS Negl. Trop. Dis. 7:e1988.

16. Eckelt, K., H. Masanas, A. Llobet, and P. Gorostiza. 2014. Automated highthroughput measurement of body movements and cardiac activity of Xenopus tropicalis tadpoles. J. Biol. Methods. 1:e9.

17. Ratcliff, W.C., J.T. Pentz, and M. Travisano. 2013. Tempo and mode of multicellular adaptation in experimentally evolved Saccharomyces cerevisiae. Evolution 67:1573-1581.

18. Wooley, C.M., R.B. Sher, A. Kale, W.N. Frankel, G.A. Cox, and K.L. Seburn. 2005. Gait analysis detects early changes in transgenic SOD1(G93A) mice. Muscle Nerve 32:43-50

19. Knippenberg, S., N. Thau, R. Dengler, and S. Petri. 2010. Significance of behavioural tests in a transgenic mouse model of amyotrophic lateral sclerosis (ALS). Behav. Brain Res. 213:82-87.

20. Alves, C.J., L.P. de Santana, A.J. dos Santos, G.P. de Oliveira, T. Duobles, J.M. Scorisa, R.S. Martins, J.R. Maximino, and G. Chadi. 2011. Early motor and electrophysiological changes in transgenic mouse model of amyotrophic lateral sclerosis and gender differences on clinical outcome. Brain Res. 1394:90-104

21. Preisig, D.F., L. Kulic, M. Kruger, F. Wirth, J. McAfoose, C. Spani, P. Gantenbein, R. Derungs, et al. 2016. High-speed video gait analysis reveals early and characteristic locomotor phenotypes in mouse models of neurodegenerative movement disorders. Behav. Brain Res. 311:340-353.
22. Van de Weerd, H.A., P.L.P. Van Loo, L.F.M. Van Zutphen, J.M. Koolhaas, and V. Baumans. 1998. Strength of preference for nesting material as environmental enrichment for laboratory mice. Appl Anim Behav Sci. 55:369-382.

23. Ambrée, O., C. Touma, N. Gortz, K. Keyvani W. Paulus, R. Palme, and N. Sachser. 2006. Activity changes and marked stereotypic behavior precede Abeta pathology in TgCRND8 Alzheimer mice. Neurobiol. Aging 27:955-964

24. Schindelin, J., I. Arganda-Carreras, E. Frise, V. Kaynig, M. Longair, T. Pietzsch, S. Preibisch, C. Rueden, et al. 2012. Fiji: an open-source platform for biological-image analysis. Nat. Methods 9:676-682.

Received 24 November 2016; accepted 06 October 2017.

Address correspondence to Hiroshi Nishimune, University of Kansas, School of Medicine, Department of Anatomy and Cell Biology, 3901 Rainbow Blvd., MS 3051, Hemenway Rm. 2073 Kansas City, KS. E-mail: hnishimune@kumc.edu

To purchase reprints of this article, contact: biotechniques@fosterprinting.com
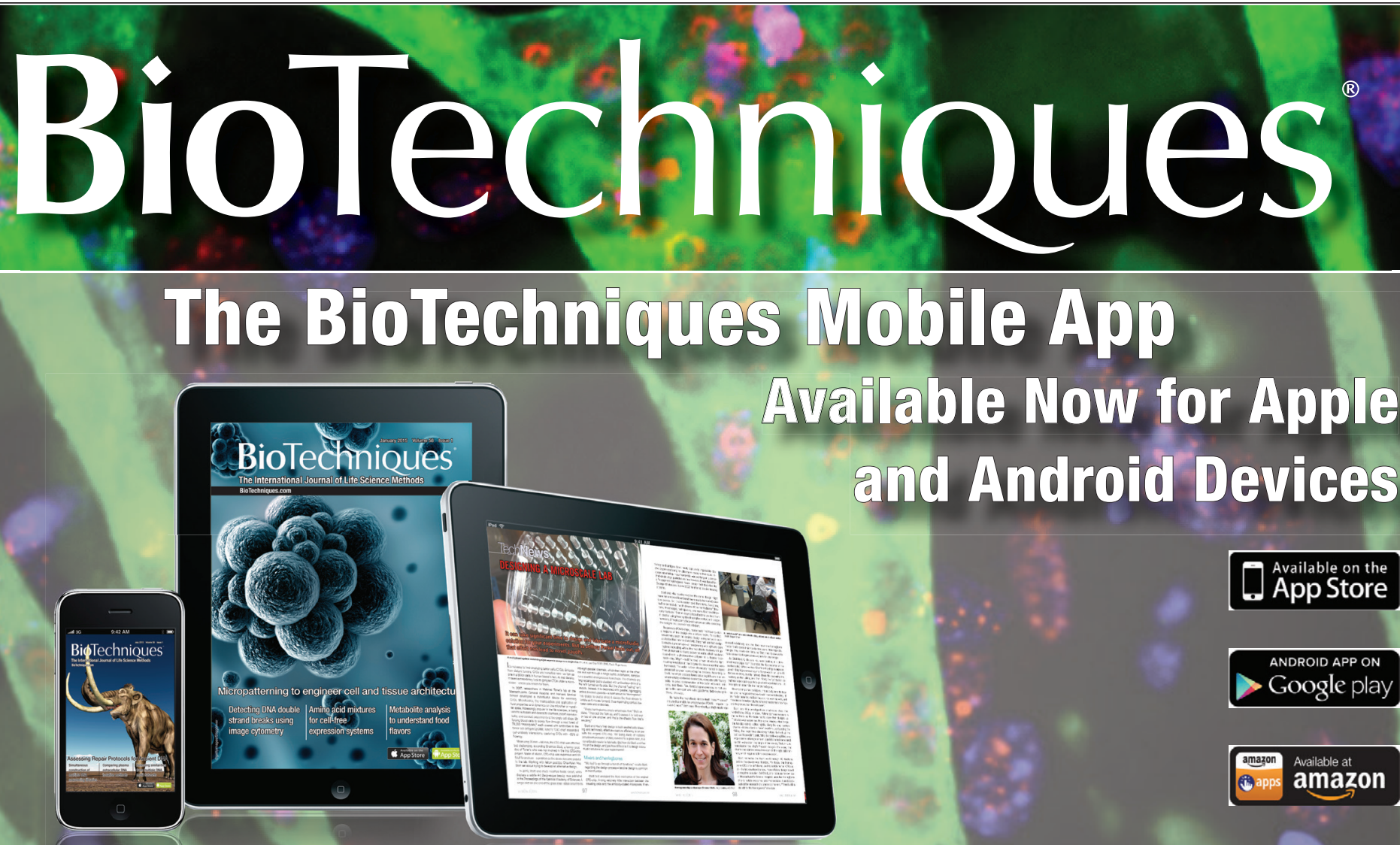

Visit BioTechniques.com/Digital to learn more and BioTechniques.com/Subscribe to receive monthly issue alerts. 